\title{
Evaluation of a radiolabeled somatostatin analog for SPECT imaging of pro-inflammatory macrophages
}

\author{
Sandra T van Tiel ${ }^{1 * *}$, Lizette Utomo ${ }^{2 *}$, Jan de Swart ${ }^{1}$, Erik de Blois ${ }^{1}$, Marion de Jong ${ }^{1}$, Yvonne M Bastiaansen-Jenniskens ${ }^{2}$ and Monique $^{R}$ \\ Bernsen $^{1}$ \\ ${ }^{1}$ Department of Radiology \& Nuclear Medicine, Erasmus MC, University Medical Center Rotterdam, the Netherlands \\ ${ }^{2}$ Department of Orthopedics, Erasmus MC, University Medical Center Rotterdam, the Netherlands
}

\begin{abstract}
Background: Macrophages are crucial in the development and progression of various diseases. To monitor their role, various proteins expressed by macrophages may be used as imaging target. In this preclinical study we investigate the value of the somatostatin receptor subtype 2 (SSTR2) as a novel imaging marker for proinflammatory macrophages, using an experimental osteoarthritis $(\mathrm{OA})$ mouse model.

Methods: SSTR2 gene expression levels in pro-inflammatory macrophages and human synovium was determined by qPCR. Tracer binding was determined in macrophages and human osteoarthritic synovium after in vitro stimulation with IFN $\gamma$ and TNF $\alpha$. Presence of pro-inflammatory macrophages in OA mice was determined by anti-CD64 $4^{+}$staining. Accumulation of the tracer in OA knees was determined by $\mu$ SPECT.

Results: Human macrophages and synovial tissue stimulated with IFN $\gamma+$ TNF $\alpha$ had significantly upregulated SSTR2 gene expression and showed increased uptake of SSTR2-targeting tracer. Shortly after OA induction an increase in the presence of pro-inflammatory macrophages was seen as assessed by immunohitochemsitry. Similar findings were obtained with SPECT, with peak uptake of the SSTR2-targeting tracer immediately after surgery followed by a gradual decrease during the course of the next 8 weeks.
\end{abstract}

Conclusions: Pro-inflammatory macrophages have elevated SSTR2 expression which makes it possible to image an inflammatory process in the knee with a radiolabeled somatostatin analog for SPECT.

\section{Introduction}

Macrophages play a crucial role in the development and progression of various diseases, for example osteoarthritis $(\mathrm{OA})[1,2]$, atherosclerotic plaque [3,4], type 2 diabetes [5,6], rheumatoid arthritis $[7,8]$ and schizophrenia $[9,10]$. Knowing the role of macrophages during disease development and progression opens possibilities for therapeutic interference. Macrophages varied roles can exacerbate and/or resolve diseases. To detect the presence of macrophage during disease processes, a specific target for imaging is needed. Depending on the activation state, macrophages express various proteins that may be used as imaging target such as the folate receptor $\beta$ [11], mannose receptor (also known as CD206) [12] and translocator protein (TSPO) [13]. These have been proposed as targets for a subtype of macrophages referred to as anti-inflammatory macrophages $[14,15]$.

Previous studies suggested that the somatostatin receptor subtype 2 (SSTR2) is also a marker for macrophages $[16,17]$. More recently, it was shown that inflammation-related uptake of an SSTR2-targeting tracer was elevated in atherosclerotic plaques and it was confirmed that the tracer had macrophage specificity [18-20]. Somatostatin is a peptide hormone which induces inhibitory effects on two key cellular processes, secretion and cell proliferation. It can bind to five distinct receptors subtypes named SSTR1, SSTR2, SSTR3, SSTR4 and SSTR5. These somatostatin receptors belong to the G-protein coupled receptor family. SSTR2 are highly expressed in human tumors, and are also present in pancreas, spleen, cerebrum, kidney, jejunum, colon, and liver $[21,22]$. Upon differentiation of human monocytes into pro-inflammatory macrophages, SSTR2 mRNA was found to be upregulated [23] in activated macrophages making it an interesting molecular target for these cells.

The aim of this preclinical study was to evaluate the potential of SSTR2 as a novel marker to monitor pro-inflammatory macrophages. We therefore determined gene expression levels of SSTR2 in unstimulated and IFN $\gamma+\mathrm{TNF} \alpha$ stimulated human macrophages and evaluated SSTR2 protein expression via binding of a STR2-binding tracer. We further used osteoarthritis (OA) as disease model to investigate a SSTR2-targeting tracer for macrophage imaging in an osteoarthritic joint. During development of OA, macrophages are attracted to the synovial lining $[24,25]$ and are pivotal in promoting the production of inflammatory and degradative mediators in the $\mathrm{OA}$ synovium, maintaining an inflammatory state in the joint. Human synovial explants were used to perform tracer binding tests in pro-inflammatory circumstances. In this study we evaluated targeting of SSTR2 as a tool for longitudinal imaging of pro-inflammatory macrophages in an experimental mouse model of OA.

${ }^{*}$ Correspondence to: S.T. van Tiel, Erasmus MC, University Medical Center Rotterdam, Department of Radiology \& Nuclear Medicine, Room Na-1120, P.O. Box 2040, 3000 CA Rotterdam, The Netherlands, Tel: +31 107044198; E-mail: s.vantiel@erasmusmc.nl

Key words: macrophage, inflammation, somatostatin receptor subtype 2, imaging, osteoarthritis

Received: June 10, 2020; Accepted: June 26, 2020; Published: July 03, 2020 


\section{Materials and methods}

\section{Ethics approval}

All animal experiments were performed in accordance with the ARRIVE guidelines for reporting on Animal Research [26], the Dutch law on animal experimentation, and were approved on March $12^{\text {th }} 2014$ by the committee on animal experimentation of the Erasmus MC, with protocol number EMC 3246. We chose male mice because sex hormones play a critical role in the progression of $\mathrm{OA}$ in the murine DMM surgical model, with males having more severe OA than females [27]. Mice were housed at the Experimental Animal Facility of the Erasmus MC with a 12-h light-dark regimen, in individually ventilated cages including extensive cage enrichment. The mice received acidified tap water and standard chow ad libitum.

Synovial tissue was obtained with implicit consent from the patients, meaning that they had the right to refuse. The protocol was approved by the medical ethical committee of the Erasmus MC on November $12^{\text {th }}$ 2004 with protocol number MEC-2004-322.

\section{Somatostatin receptor subtype 2 expression}

To verify the expression of SSTR2 on monocyte-derived macrophages, primary human monocytes were isolated from five buffy coats of male donor blood samples $(58 \pm 8 \mathrm{Y}$; Sanquin Blood bank, Amsterdam, the Netherlands) by Ficoll density gradient separation (GE Healthcare, Little Chalfont, UK) and $\mathrm{CD} 14^{+}$magneticactivated- cell sorting (MACS; Miltenyi, Bergisch Gladbach, Germany), as previously described [15]. To acquire pro-inflammatory macrophages in vitro $[15,28]$, the monocytes were stimulated $1 \mathrm{~h}$ after plating with $10 \mathrm{ng} / \mathrm{mL}$ IFN- $\gamma$ (PeproTech, New Jersey, USA) and $10 \mathrm{ng} / \mathrm{mL}$ TNF- $\alpha$ (PeproTech, New Jersey, USA) in X-VIVO 15 medium (Lonza, Verviers, Belgium) supplemented with $20 \%$ heat-inactivated fetal calf serum (FCS; Lonza, Verviers, Belgium), $50 \mu \mathrm{g} / \mathrm{mL}$ gentamicin (Gibco, Carlsbad, USA) and $1.5 \mu \mathrm{g} / \mathrm{mL}$ amphotericin B (Gibco, Carlsbad, USA), from now on referred to as $\mathrm{M}(\mathrm{IFN} \gamma+\mathrm{TNF} \alpha)$. The macrophages were stimulated 3 days at $37^{\circ} \mathrm{C}$ and $5 \% \mathrm{CO}_{2}$. Media and stimuli were refreshed $24 \mathrm{~h}$ prior to harvest.

To determine the expression of SSTR2 in human synovial tissue, synovium was obtained as waste material from OA patients $(n=4$, $60 \pm 13 \mathrm{Y}$ ) undergoing total knee replacement surgery. Fat tissue was macroscopically removed and the synovium was cut into pieces of approximately $40 \mathrm{mg}$ wet weight and cultured in Dulbecco's Modified Eagle Medium, low glucose (DMEM; Gibco, Carlsbad, USA), supplemented with $1 \%$ Insulin-Transferrin-Selenium (ITS+ Premix, Corning, New York, USA), $50 \mu \mathrm{g} / \mathrm{mL}$ gentamicin (Gibco, Carlsbad, USA), $1.5 \mu \mathrm{g} / \mathrm{mL}$ amphotericin B (Fungizone; Gibco, Carlsbad, USA) and $25 \mu \mathrm{g} / \mathrm{mL}$ L-ascorbic acid 2-phosphate (Sigma-Aldrich, St. Louis, USA). To simulate acute inflammation, half of the number of the explants was stimulated with $10 \mathrm{ng} / \mathrm{mL}$ IFN $\gamma+10 \mathrm{ng} / \mathrm{mL}$ TNFa. After $24 \mathrm{~h}$ of stimulation, the synovial explants were harvested and stored at $-80^{\circ} \mathrm{C}$ until gene expression analysis.

Messenger RNA (mRNA) was isolated from the macrophages using the RNeasy Micro Kit (Qiagen, Hilden, Germany) according to manufacturer's instructions. For the synovial explants, the tissue was first frozen in liquid nitrogen followed by pulverization using a MikroDismembrator (B. Braun Biotech International $\mathrm{GmbH}$, Melsungen, Germany) at $3000 \mathrm{rpm}$. The tissue was then homogenized with Trizol (Gibco, Carlsbad, USA) and 20\% chloroform (Sigma-Aldrich, St. Louis, USA). Complementary DNA (cDNA) was synthesized using the RevertAid $^{\text {ma }}$ First Strand cDNA Synthesis Kit (Fermentas GmbH, Leon-
Rot, Germany) according to manufacturer's instructions. Expression of SSTR2 was evaluated using a TaqMan SSTR2 Gene Expression Assay (Hs00990356_m1; Thermo Fisher Scientific). Glyceraldehyde3-phosphate dehydrogenase (GAPDH; Fw: CAACGGATTTGGT CGTATTGGG; Rev: TGCCATGGGTGGAATCATATTGG; Probe: GGCGCCCCAACCAGCC) was used as a housekeeping gene.

\section{Radiolabeled tracers}

Two SSTR2 agonists, DOTA-Tyr ${ }^{3}$-octreotate, further referred to as DOTA-TATE (BioSynthema, Saint Louis, USA) and DTPA-octreotide (Mallinckrodt Medical, Petten, The Netherlands), and the SSTR2 antagonist DOTA-JR11 (kindly provided by Dr. Helmut Maecke) were used. The tracers were radiolabeled with Indium-111 (Covidien, Zaltbommel, The Netherlands) as previously described [29,30]. Molar activity was $200-300 \mathrm{MBq} / \mathrm{nmol}$. Radiochemical yield was $>99 \%$ and radiochemical purity was $>90 \%$ for each tracer.

\section{Binding of SSTR2 tracer}

The binding of the radiotracer to SSTR2 present on macrophages was established in vitro. Human monocytes were seeded in 6-well plates at a density of $5 \times 10^{5} \mathrm{cells} / \mathrm{cm}^{2}$, and subsequently cultured for 3 days with IFN $\gamma$ and TNFa, as described in the previous paragraph. After 3 days of stimulation, the macrophages were washed with PBS (Gibco, Carlsbad, USA) and were incubated with $4 \times 10^{-9} \mathrm{M}\left[{ }^{111} \mathrm{In}\right]$ In-DOTATATE. To determine specific binding the cells were also incubated with $4 \times 10^{-9} \mathrm{M}$ [ $\left.{ }^{111} \mathrm{In}\right]$ In-DOTA-TATE plus $10^{-6} \mathrm{M}$ DOTA-TATE for $1 \mathrm{~h}$ at $37^{\circ} \mathrm{C}$. Cellular uptake of the tracer was stopped by removing the supernatant and washing twice with cold PBS. The cells were lysed with $0.1 \mathrm{M} \mathrm{NaOH}$ and the amount of radioactivity present in the samples was counted in a gamma counter (1480 WIZARD automatic gamma counter, PerkinElmer, Turku, Finland).

In vitro autoradiography was performed on human OA synovium cryosections $(10 \mu \mathrm{m})$. The sections were incubated for 1 hour with $80 \mu \mathrm{L}$ $10^{-9} \mathrm{M}\left[{ }^{111} \mathrm{In}\right] \mathrm{In}$-DOTA-JR11 with or without excess $\left(10^{-6} \mathrm{M}\right)$ unlabeled DOTA-TATE to determine non-specific binding. After incubation, the excess radiotracer was removed and the sections were exposed to super resolution phosphor screens (Packard Instruments Co., Meriden, USA) for 7 days. Binding of the radiotracers to SSTR2 containing areas in the sections was quantified using Optiquant (Packard Instruments Co., Groningen, The Netherlands) and expressed as density light units/ $\mathrm{mm}^{2}\left(\mathrm{DLU} / \mathrm{mm}^{2}\right)$. Sections of human $\mathrm{H} 69$ tumour, an SSTR2-positive xenograft, were used as positive control. All sections were stained with hematoxylin and eosin to determine the localization of cell dense areas where the macrophages can be found.

\section{Osteoarthritis induction}

Experimental OA was induced, during light time of the day/ night cycle, by destabilization of the medial meniscus (DMM) [31]. The right knees of 40 male C57BL/6 mice of 12-14 weeks old (Harlan Laboratories/Envigo, Cambridgeshire, UK) underwent dissection of the medial meniscotibial ligament (MMTL). As control, the contralateral knee underwent a sham procedure, which entailed no transection of the MMTL.

\section{Immunohistochemical analysis}

At $1,3,7,14,28$, and 56 days after OA induction both DMM and control knees were excised and fixed for 10 days in $4 \%$ formaldehyde (BoomLab, Meppel, The Netherlands). After decalcification the knees 
were dehydrated, embedded in paraffin and completely sectioned in the coronal plane. For the CD64 histochemical (IHC) staining, a CD64 antibody (Sino-Biological, 50086-R001, $5 \mu \mathrm{g} / \mathrm{ml}$ ) was incubated on the slides for $60 \mathrm{~min}$, and subsequently incubated with link and label (Concentrated MultiLink ${ }^{\diamond}$ and Concentrated AP Label; BioGenex, Fremont, California, USA); New Fuchsine was used as substrate [32]. The extent of positive CD64 staining in the sections was scored by bright- field microscopy and ranked. Ranking means that all knees were arranged from least intensely stained to most intensely stained using bright field microscopy. The staining intensity of the markers was ranked amongst all time points.

To confirm that OA was induced after 8 weeks, three consecutive thionin-stained sections of the medial femoral condyle and medial tibial plateau were evaluated. The structural cartilage damage was scored by the OARSI scoring system by Glasson et al. [1,33]. The score of the quadrants of three consecutive sections was summed and used for analysis. Histological images were acquired with a NanoZoomer 2.0-HT slide scanner (Hamamatsu, Hamamatsu City, Japan).

\section{In vivo imaging}

To evaluate the influx of pro-inflammatory macrophages during OA development, OA was induced in mice $(n=6)$ as described above. Because macrophage polarization is a dynamic process, we wanted to scan the knee as soon as possible after tracer injection. The mice were imaged 2 hours after i.v. injection of radiolabeled tracer (60 $\mathrm{MBq} / 200$ pmol [111 In]In-DTPA-octreotide) on day $1,3,7,14,28$ or 56 after induction of OA. At day 3 after OA induction 3 mice were injected with $40 \mathrm{nmol}$ block, DOTA-Tyr ${ }^{3}$-TATE, 1 minute prior to an injection with $60 \mathrm{MBq} / 200 \mathrm{pmol}\left[{ }^{111} \mathrm{In}\right] \mathrm{In}-\mathrm{DTPA}$ - octreotide. Singlephoton emission computed tomography (SPECT) was performed on a MILabs VECTor+/CT system (Utrecht, the Netherlands) [34] with an ultra-high sensitivity mouse collimator with fifty-four $2.0 \mathrm{~mm}$ pinholes. A 55-minutes SPECT was acquired in list mode along a spiral trajectory, scan mode fine and 10 positions. SPECT was followed by CT ( $55 \mathrm{keV}, 615 \mathrm{~mA})$. SPECT data were reconstructed using the pixelbased ordered subsets expectation maximization (POSEM) developed by MILabs [35] algorithm with 30 iterations, 4 subsets and a voxel size of $0.4 \mathrm{~mm}$. Photopeaks 171 and $245 \mathrm{keV}$ were used, scatter and background were corrected for with the triple-energy-window method (for $171 \mathrm{keV}$ peak \% width of 7 and for $245 \mathrm{keV}$ peak \% width of 10 )

A

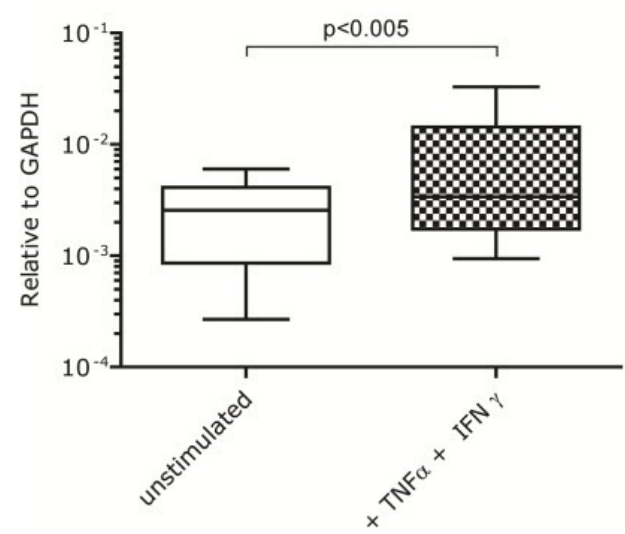

[36]. CT was reconstructed using filtered back-projection. SPECT and CT were registered; SPECT data were corrected for attenuation using the CT data [34]. A post-reconstruction 3- dimensional Gaussian filter was applied. The resulting data was analyzed using inviCRO VivoQuant software (Boston, MA, USA) by quantification of manually drawn regions of interests (ROIs) based on CT. All data were represented as percentage of the injected dose per tissue volume $\% \mathrm{IA} / \mathrm{cm}^{3}$ ).

\section{Statistical analysis}

For the in vitro experiments, a mixed linear model after log transform was used followed by a Bonferroni's post hoc comparisons test using PASW Statistics 22.0 (SPSS Inc. Chicago, USA). For the histological staining experiment, differences in CD64 ranks for the time points were determined by a Kruskal-Wallis test. In the uptake experiment and autoradiography the mean values between the different groups ( \pm stimulation, \pm block) were compared and analyzed with a Two-way ANOVA. To compare values for DMM and sham surgery (in vivo imaging) the paired $t$-test was applied. Differences were considered statistically significant when $p<0.05$.

\section{Results}

\section{Somatostatin receptor subtype 2 mRNA expression}

To establish the effect of inflammatory stimuli on SSTR2 mRNA expression of macrophages, SSTR2 mRNA levels were determined in unstimulated and IFN $\gamma+\mathrm{TNF} \alpha$-stimulated macrophages by qPCR. Following stimulation with IFN $\gamma+\mathrm{TNF}$, expression of SSTR2 mRNA significantly increased by a factor of $3.6(p<0.005)$ relative to unstimulated macrophages (Figure 1A). Similar to macrophages, stimulation of synovial tissue of OA patients with IFN $\gamma+\mathrm{TNF} \alpha$, also resulted in increased expression levels of SSTR2 mRNA (10-fold, $p<0.001)$ relative to unstimulated tissue (Figure 1B).

\section{Binding of SSTR2 tracer}

Stimulation of primary monocyte-derived macrophages with IFN $\gamma+\mathrm{TNFa}$ in vitro resulted in a significant increase of $\left[{ }^{111} \mathrm{In}\right] \mathrm{In}$ DOTA-TATE uptake (Figure 2). Relative to unstimulated macrophages the uptake of $\left[{ }^{111} \mathrm{In}\right]$ In-DOTA-TATE increased (1.6-fold, $\left.p<0.05\right)$. For both stimulated and unstimulated macrophages uptake of [ $\left.{ }^{111} \mathrm{In}\right]$ In-DOTA-TATE was largely blocked following addition of excess unlabeled DOTA-TATE $(p<0.001)$.

B

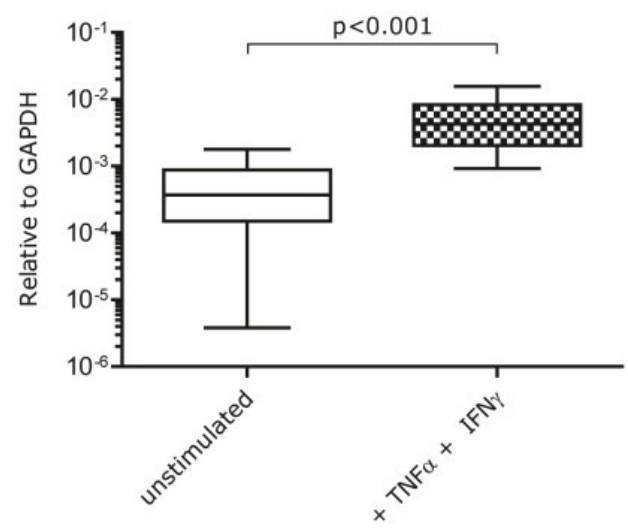

Figure 1. Gene expression levels of somatostatin receptor subtype 2 (SSTR2) in human primary macrophages and osteoarthritic synovial tissue. SSTR2 gene expression relative to the expression of GAPDH in (A) unstimulated human monocyte-derived macrophages and IFN $\gamma+\mathrm{TNF} \alpha$-stimulated macrophages. (B) osteoarthritic synovial tissue, cultured in vitro, with and without IFN $\gamma+\mathrm{TNF} \alpha$ stimulation. Data is presented from min to max including the median. 


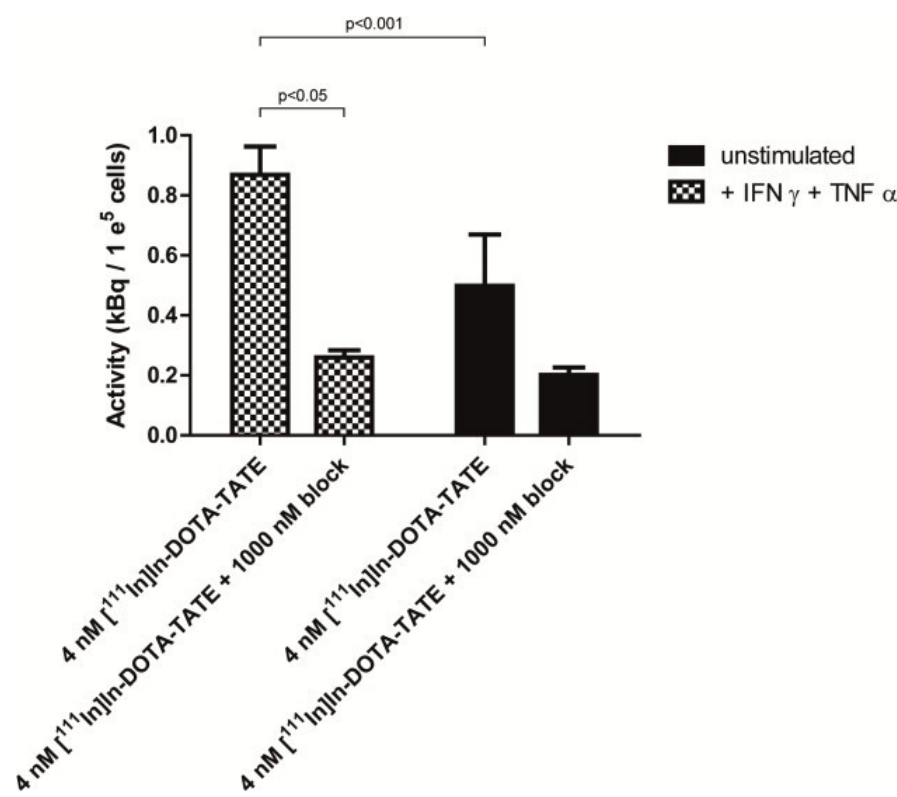

Figure 2. Binding of [111In]In-DOTA-TATE to SSTR2 on human monocyte-derived macrophages. Binding of $\left[{ }^{111}\right.$ In]In-DOTA-TATE (as a measure of SSTR2 presence) to $\mathrm{M}(\mathrm{IFN} \gamma+\mathrm{TNF} \alpha)$ in $\mathrm{kBq} / 1 \mathrm{e}^{5}$ cells. Blocking of the specific binding to SSTR 2 was performed with excess of unlabeled DOTA-TATE. Data is presented as mean $+\mathrm{SD}$.

Autoradiography analysis of binding of the radiolabeled tracer [111In]In-DOTA-JR11 showed regions with higher signal intensities corresponding to cell dense areas on H\&E-stained sections (Figures 3A and $3 \mathrm{~B}$ ). The intensity of the autoradiography signal was significantly increased up to 3.1 times (average 1.6 ; range $0.5-3.1 ; p<0.05$ ) in IFN $\gamma+\mathrm{TNF} \alpha$-stimulated synovial tissue relative to unstimulated synovial tissue (Figure 3C).

\section{Presence of macrophages during experimental OA}

To establish the relevance of SSTR2 tracer as a marker for proinflammatory macrophages in vivo, presence of macrophages and uptake of SSTR2 tracer was studied in a mouse DMM model for OA over time.

From knees, which were harvested eight weeks after induction of OA by DMM, three consecutive thionin-stained sections of the medial femoral condyle and medial tibial plateau were evaluated. The summed OARSI scores for three DMM knees were: $2.5,4.0$, and 10.5. Osteophytes were present in all DMM knees. Control knees did not have any signs of cartilage degradation (OARSI score 0 for all knees) and did not present any indications of osteophyte formation (Figure 4).

CD64 positive cells, indicating pro-inflammatory macrophages, were present from day 1 onward. The sections were ranked to distinguish low-positivity and high-positivity. The number of $\mathrm{CD} 64^{+}$macrophages were elevated at day 1 and 3 , and slowly diminished during the following weeks. There were statistically differences seen between the ranks at the different time points $(p<0.0001)$. No statistically significant differences were seen in the pattern of $\mathrm{CD}_{64}^{+}$macrophages presence over time between the sham-operated and DMM knees (Figure 5).

In Figure 6A a representative SPECT/CT image is shown of a DMM knee 3 days after destabilization of the medial meniscus in which accumulation of the radioactive tracer was observed. Percentage injected activity per volume $\left(\% \mathrm{IA} / \mathrm{cm}^{3}\right)$ was calculated over time after DMM. During the first 7 days after DMM surgery, increased amounts of radioactivity were found in the knees, which gradually decreased over time (Figure 6B). Herein we saw a similar pattern for both the knees in which OA was induced by DMM and for the knees that underwent sham surgery.

To determine the specificity of the radioactive signal a blocking experiment was executed. After the SPECT/CT scans the knees and
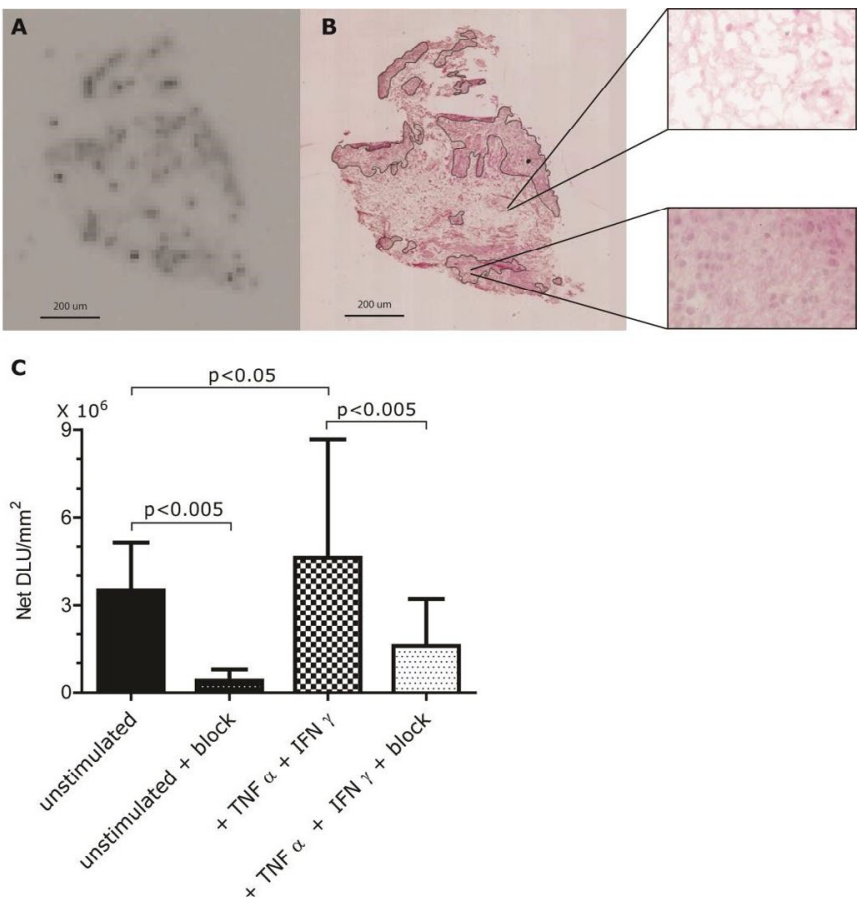

Figure 3. Binding of [ $\left.{ }^{111} \mathrm{In}\right]$ In-DOTA-JR 11 to human synovial tissue. (A) Autoradiography of a section of an OA synovial explant. Dark spots indicate uptake of [ ${ }^{111}$ In]In-DOTA-JR11. (B) H\&E staining of IFN $\gamma+\mathrm{TNF} \alpha$-stimulated synovial tissue of the same sample shown in A with $240 X$ magnifications. Segmentation lines indicate relevant areas used for analysis. (C) Binding of [ $\left.{ }^{111} \mathrm{In}\right] \mathrm{In}-\mathrm{DOTA}-J R 11$ to human synovial tissue is shown as NET DLU/mm² + SD. Samples of synovium derived from 3 different OA patients undergoing total knee resection were incubated with (two right bars) and without IFNy/TNF $\alpha$ (two left bars). To determine specific binding a 1000-times excess of unlabeled DOTA-TATE was added to some samples (solid black bars).
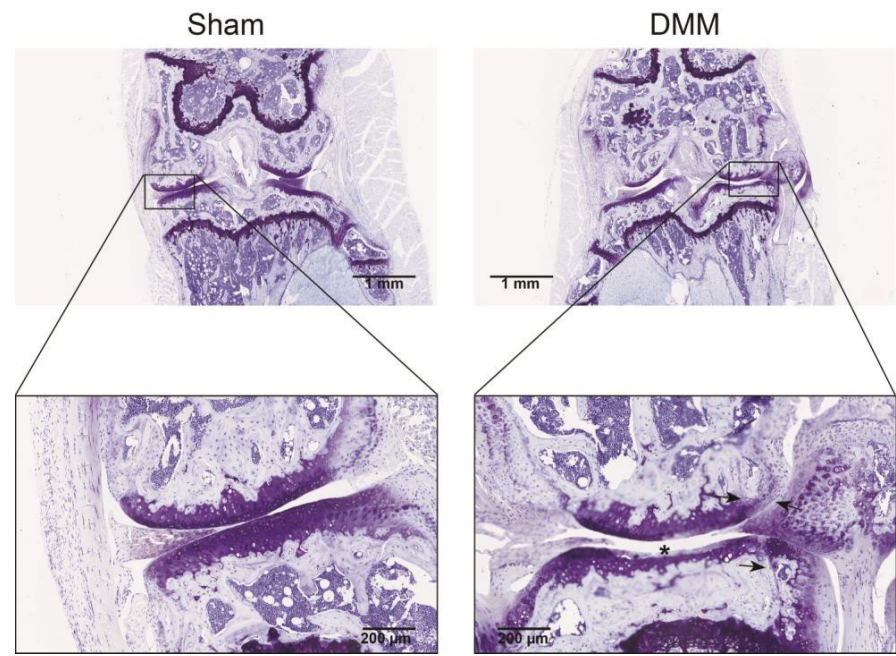

Figure 4. Cartilage damage and osteophyte formation after induction of experimental osteoarthritis (OA) by DMM. Thionin staining of a mouse knee 8 weeks after OA induction by destabilization of the medial meniscus (DMM). The femur condyle and tibia plateau are shown in the enlargements. The sham-operated knee had a summed OARSI score of 0 and the knee with DMM had a summed OARSI score of 10.5 (maximum possible score 72 ). Cartilage damage (at asterisk) and osteophytes (indicated by the arrows) were seen in all DMM knees. 


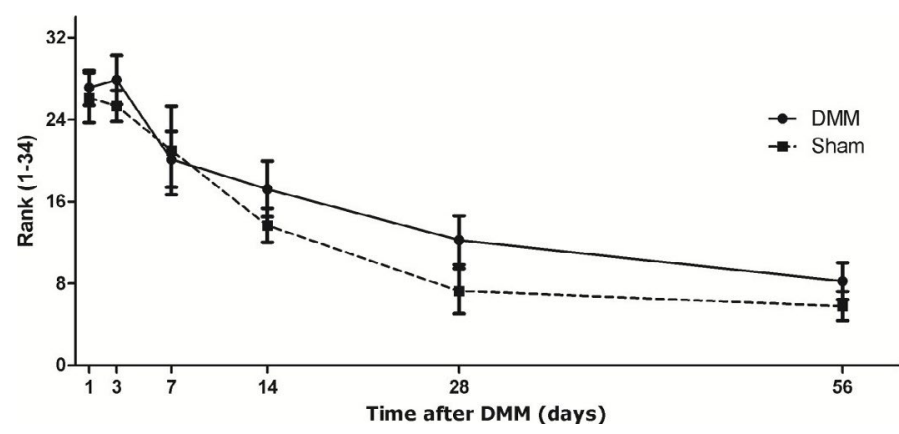

Figure 5. Presence of CD64+ macrophages during development of experimental osteoarthritis after DMM. The continuous line represents the DMM knees. The dotted line represents the sham knees. Each group contains 7-9 knees. Data is presented as mean $\pm \mathrm{SD}$, $p<0.0001$ between different time points.

A
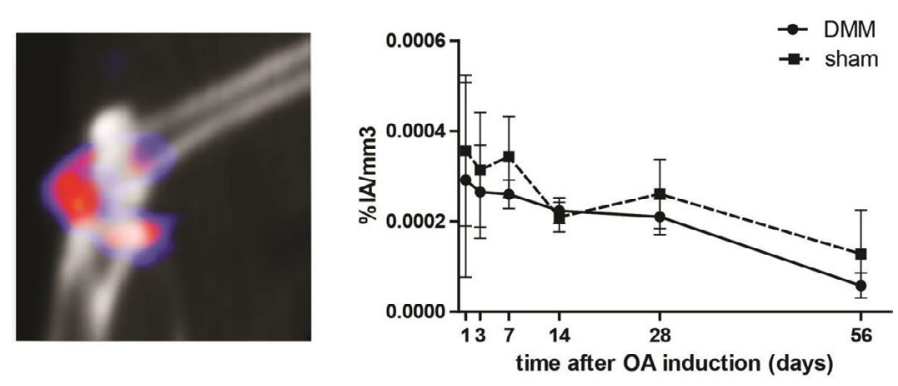

Figure 6. SPECT/CT imaging of in vivo uptake of [111In]In-DTPA-octreotide in osteoarthritic (OA) mice knees. (A) Sagittal view of a knee 3 days after OA induction through destabilization of the medial meniscus (DMM). Scan was made $2 \mathrm{~h}$ post injection with [ $\left.{ }^{111} \mathrm{In}\right]$ In-DTPA-octreotide. (B) Percentage injected activity ([ $\left.{ }^{111} \mathrm{In}\right] \mathrm{In}-\mathrm{DTPA}$ octreotide) per volume (knee) $\left(\% \mathrm{IA} / \mathrm{cm}^{3}\right)$ was calculated over time after induction of OA The continuous line represents the DMM knees. The dotted line represents the sham knees. Each group contains 6 animals. Data is presented as mean $\pm \mathrm{SD}, p=0.099$.

thymus were removed and placed in a gammacounter. Both SPECT/CT scans and gammacounter data showed that the binding was receptorspecific (Supplementary Digital Material 1: Supplementary Figure 1). Co-injection of excess unlabeled DOTA- Tyr ${ }^{3}$-TATE reduced uptake of $\left[{ }^{111} \mathrm{In}\right]$ In-DTPA-octreotide in the knees and thymus.

\section{Discussion}

Pro-inflammatory macrophages have been identified as crucial mediators in various disease processes. Non-invasive monitoring of their presence during disease progression is therefore very desirable. In this report, we showed that pro-inflammatory macrophages (IFN $\gamma+$ TNF $\alpha$ stimulated) had elevated SSTR2 expression and showed concomitant increased uptake of SSTR2-targeting radiolabeled peptides, which are suitable as SPECT tracers. Using such a SSTR2 SPECT tracer in a translational setting, we also demonstrated that under pro-inflammatory conditions there was increased binding of the tracer in human OA synovium. Moreover, in an in vivo model we showed that macrophage-associated inflammation resulted in concomitant uptake of [ $\left.{ }^{111} \mathrm{In}\right]$ In-DTPA- octreotide in mouse knees, indicating that an SSTR2-targeting SPECT tracer can be used to monitor the course of a pro-inflammatory response over time in vivo.

Destabilization of the medial meniscus in mice was used as a model for OA with anticipated involvement of pro-inflammatory macrophages. In many other studies this model has been used for studying processes during the onset of $\mathrm{OA}$ and in studies evaluating novel intervention strategies for OA [37-39]. This animal model has a low variability
[31] and represents posttraumatic human injury which leads to joint degeneration [40]. During human OA development a macrophage influx was seen [41] and macrophage-associated inflammation was related with structural damage and disease progression.

As shown in this study, it appeared that the influx of macrophages was time dependent and associated with the development path of OA, but the macrophage influx in the knee was not exclusively associated with development of OA. An influx of macrophages was also observed in the sham-operated knee, most likely due to the inflammatory reaction to tissue damage caused by surgical manipulation. It should be noted that the sham knees had no structural cartilage damage or osteophyte formation, features that are associated with OA. Since the SSTR2 tracer specifically targets inflammatory cells, and not damaged cartilage, SSTR2 receptor uptake occurred in both DMM and shamoperated knees due to the presence of inflammatory macrophages in both situations. The pattern seen with uptake of SSTR2 targeting tracer in the knee, and the pattern seen with the presence of pro-inflammatory macrophages (histological aCD64 staining) of the knee, proceeded in the same way; starting with an high influx of macrophages which decreased during the course of time.

For potential clinical relevance we evaluated SSTR2 tracer uptake in synovial tissue samples derived from OA patients. OA synovium showed specific uptake of [ $\left.{ }^{111} \mathrm{In}\right]$ In-DOTA-JR11, which could be further enhanced under acutely induced inflammatory conditions (stimulation with IFN $\gamma+\mathrm{TNF} \alpha$ ). There was considerable variation in uptake of the SSTR2 tracer between patient samples which was most likely due to the large heterogeneity in disease state between OA patients and whether or not a given patient had a flare of inflammation at time of surgery. It is also known that synovium consists of a heterogeneous cell population including synoviocytes, macrophages and fibroblasts. Although it cannot fully be excluded that SSTR2-binding tracer is taken up by cells other than macrophages, we consider this option highly unlikely to substantially contribute to the measured activity. It is known that the number of macrophages dramatically increases in inflammatory arthritis relative to healthy synovia [41,42], also seen in this study using histological evaluation. It is therefore most likely that the signal in inflamed knees is caused by the presence of pro-inflammatory macrophages.

In this research high resolution scans are of utmost importance, because the target is very small. That is why we used an indium-111 labeled tracer. When this tracer is going to be used in the clinic we will be using a radioactive isotope compatible with PET. PET has in the clinic a higher resolution, half-life of radioactive isotopes is shorter so patients can leave the hospital sooner and images are less noisy therefor accurate quantification can be performed.

We made use of a mouse model where we introduced OA through destabilization of the medial meniscus. Mice knees are very small and DMM results in a mild inflammation. Although we had these restrictions, we were able to successfully visualize, quantify and monitor the inflammation process by SPECT/CT imaging. Perhaps with the use of a larger animal model and/or a disease model with more macrophage involvement, like rheumatoid arthritis [43,44], will facilitate further research. Our findings are promising for the use of SSTR2 targeting tracers in studying macrophage involvement in disease processes. Macrophages are crucial in the development and progression of various diseases and that is why accurate imaging of pro-inflammatory macrophages, beside the already established antiinflammatory macrophage tracers $[11,45]$, might be an important step 
forward in understanding disease development. This is also indicated by recent publications on studies in which macrophages are being imaged in atherosclerotic plaques using the folate, mannose and somatostatine type 2 receptor $[18,46,47]$.

\section{Conclusion}

Our research showed that pro-inflammatory macrophages (IFN $\gamma+\mathrm{TNF} \alpha$ stimulated) had elevated SSTR2 expression and show concomitant elevated binding of SSTR2-targeting radiolabeled peptides suitable as SPECT tracers. Under pro-inflammatory conditions there was an increase in presence of $\mathrm{CD}^{+} 4^{+}$cells especially at day 1 and 3 after OA induction. In vivo SSTR2 SPECT imaging showed an increase of radioactivity in the knee at day 1-7 after OA induction. So, SSTR2 can be a marker to longitudinally monitor pro-inflammatory macrophages in vivo.

\section{Conflicts of interest}

There are no conflicts of interest to be declared.

\section{Acknowledgements}

All imaging experiments were conducted at the Applied Molecular Imaging at Erasmus MC Facility (AMIE; https://www.erasmusmc.nl/ en/research/core-facilities/amie). This research was done within the postgraduate school Molecular Medicine, Erasmus MC, University Medical Center, The Netherlands. The authors thank Joost Haeck, Gabriela Doeswijk, Mirjam Pikaart and Nicole Kops for their expert assistance during the studies and Mark Konijnenberg for his statistical analysis of the data.

\section{References}

1. Blom AB, van Lent PL, Holthuysen AE, van der Kraan PM, Roth J, et al. (2004) Synovial lining macrophages mediate osteophyte formation during experimental osteoarthritis. Osteoarthritis Cartilage. 12: 627-635. [Crossref]

2. Lopes EBP, Filiberti A, Husain SA, Humphrey MB (2017) Immune Contributions to Osteoarthritis. Curr Osteoporos Rep 15: 593-600. [Crossref]

3. Moore KJ, Sheedy FJ, Fisher EA (2013) Macrophages in atherosclerosis: a dynamic balance. Nat Rev Immunol 13: 709-721. [Crossref]

4. Colin S, Chinetti-Gbaguidi G, Staels B (2014) Macrophage phenotypes in atherosclerosis. Immunol Rev. 262: 153-166. [Crossref]

5. Kraakman MJ, Murphy AJ, Jandeleit-Dahm K, Kammoun HL (2014) Macrophage polarization in obesity and type 2 diabetes: weighing down our understanding of macrophage function? Front Immunol 5: 470. [Crossref]

6. Xu H, Barnes GT, Yang Q, Tan G, Yang D, et al. (2003) Chronic inflammation in fat plays a crucial role in the development of obesity-related insulin resistance. $J$ Clin Invest 112: 1821-1830. [Crossref]

7. Laria A, Lurati A, Marrazza M, Mazzocchi D, Re KA, et al. (2016) The macrophages in rheumatic diseases. J Inflamm Res 9: 1-11. [Crossref]

8. Zhang Q, Yuan R, Li C, Wei W, Shen W, et al. (2019) Macrophage depletion with clodronate-containing liposomes affects the incidence and development of rheumatoid arthritis. Z Rheumatol 78: 996-1003. [Crossref]

9. Takahashi Y, Yu Z, Sakai M, Tomita H (2016) Linking Activation of Microglia and Peripheral Monocytic Cells to the Pathophysiology of Psychiatric Disorders. Front Cell Neurosci 10: 144. [Crossref]

10. Frick LR, Williams K, Pittenger C (2013) Microglial dysregulation in psychiatric disease. Clin Dev Immunol. 2013: 608654

11. Puig-Kroger A, Sierra-Filardi E, Dominguez-Soto A, Samaniego R, Corcuera MT, et al. (2009) Folate receptor beta is expressed by tumor-associated macrophages and constitutes a marker for M2 anti-inflammatory/regulatory macrophages. Cancer Res 69: 9395-9403. [Crossref]

12. Bala G, Baudhuin H, Remory I, Gillis K, Debie P, et al. (2018) Evaluation of [(99m) Tc]Radiolabeled Macrophage Mannose Receptor-Specific Nanobodies for Targeting of Atherosclerotic Lesions in Mice. Mol Imaging Biol 20: 260-267. [Crossref]
13. Narayan N, Mandhair H, Smyth E, Dakin SG, Kiriakidis S, et al. (2017) The macrophage marker translocator protein (TSPO) is down-regulated on pro-inflammatory 'M1' human macrophages. PLoS One 12: e0185767. [Crossref]

14. Van De Wiele C, Sathekge M, Maes A (2014) Targeting monocytes and macrophages by means of SPECT and PET. Q J Nucl Med Mol Imaging 58: 269-275. [Crossref]

15. Utomo L, van Osch GJ, Bayon Y, Verhaar JA, Bastiaansen-Jenniskens YM (2016) Guiding synovial inflammation by macrophage phenotype modulation: an in vitro study towards a therapy for osteoarthritis. Osteoarthritis Cartilage 24: 1629-1638. [Crossref]

16. ten Bokum AM, Hofland LJ, van Hagen PM (2000) Somatostatin and somatostatin receptors in the immune system: a review. Eur Cytokine Netw 11: 161-176. [Crossref]

17. Dalm VA, van Hagen PM, van Koetsveld PM, Achilefu S, Houtsmuller AB, et al (2003) Expression of somatostatin, cortistatin, and somatostatin receptors in human monocytes, macrophages, and dendritic cells. Am J Physiol Endocrinol Metab 285: E344-E353. [Crossref]

18. Tarkin JM, Joshi FR, Evans NR, Chowdhury MM, Figg NL, et al. (2017) Detection of Atherosclerotic Inflammation by (68)Ga-DOTATATE PET Compared to [(18)F]FDG PET Imaging. J Am Coll Cardiol 69: 1774-1791. [Crossref]

19. Rominger A, Saam T, Vogl E, Ubleis C, la Fougere C, et al. (2010) In vivo imaging of macrophage activity in the coronary arteries using 68Ga-DOTATATE PET/CT: correlation with coronary calcium burden and risk factors. J Nucl Med 51: 193-197. [Crossref]

20. Li X, Bauer W, Kreissl MC, Weirather J, Bauer E, et al. (2013) Specific somatostatin receptor II expression in arterial plaque: (68)Ga-DOTATATE autoradiographic, immunohistochemical and flow cytometric studies in apoE-deficient mice. Atherosclerosis 230: 33-39. [Crossref]

21. Patel YC (1999) Somatostatin and its receptor family. Front Neuroendocrinol 20: 157 198. [Crossref]

22. Gunther T, Tulipano G, Dournaud P, Bousquet C, Csaba Z, et al. (2018) International Union of Basic and Clinical Pharmacology. CV. Somatostatin Receptors: Structure, Function, Ligands, and New Nomenclature. Pharmacol Rev 70: 763-835. [Crossref]

23. Armani C, Catalani E, Balbarini A, Bagnoli P, Cervia D (2007) Expression, pharmacology, and functional role of somatostatin receptor subtypes 1 and 2 in human macrophages. J Leukoc Biol 81: 845-855. [Crossref]

24. Benito MJ, Veale DJ, FitzGerald O, van den Berg WB, Bresnihan B (2005) Synovial tissue inflammation in early and late osteoarthritis. Ann Rheum Dis 64: 1263-1267. [Crossref]

25. Ene R, Sinescu RD, Ene P, Cirstoiu MM, Cirstoiu FC (2015) Synovial inflammation in patients with different stages of knee osteoarthritis. Rom J Morphol Embryol 56: 169-173. [Crossref]

26. Kilkenny C, Browne WJ, Cuthill IC, Emerson M, Altman DG (2010) Improving bioscience research reporting: the ARRIVE guidelines for reporting animal research. PLoS Biol 8: e1000412. [Crossref]

27. Ma HL, Blanchet TJ, Peluso D, Hopkins B, Morris EA, et al. (2007) Osteoarthritis severity is sex dependent in a surgical mouse model. Osteoarthritis Cartilage 15: 695700. [Crossref]

28. Utomo L, Bastiaansen-Jenniskens YM, Verhaar JA, van Osch GJ (2016) Cartilage inflammation and degeneration is enhanced by pro-inflammatory (M1) macrophages in vitro, but not inhibited directly by anti-inflammatory (M2) macrophages. Osteoarthritis Cartilage 24: 2162-2170. [Crossref]

29. de Blois E, Chan HS, de Zanger R, Konijnenberg M, Breeman WA (2014) Application of single-vial ready-for-use formulation of $111 \mathrm{In}$ - or $177 \mathrm{Lu}$-labelled somatostatin analogs. Appl Radiat Isot 85: 28-33. [Crossref]

30. Bakker WH, Albert R, Bruns C, Breeman WA, Hofland LJ, et al. (1991) [111In-DTPAD-Phe1]-octreotide, a potential radiopharmaceutical for imaging of somatostatin receptor-positive tumors: synthesis, radiolabeling and in vitro validation. Life Sci 49: 1583-1591. [Crossref]

31. Glasson SS, Blanchet TJ, Morris EA (2017) The surgical destabilization of the medial meniscus (DMM) model of osteoarthritis in the 129/SvEv mouse. Osteoarthritis Cartilage 15: 1061-1069. [Crossref]

32. Gautier EL, Shay T, Miller J, Greter M, Jakubzick C, et al. (2012) Gene-expression profiles and transcriptional regulatory pathways that underlie the identity and diversity of mouse tissue macrophages. 13: 1118-1128. [Crossref]

33. Glasson SS, Chambers MG, Van Den Berg WB, Little CB (2010) The OARSI histopathology initiative - recommendations for histological assessments of osteoarthritis in the mouse. Osteoarthritis Cartilage 18 Supp1 3: S17-S23. [Crossref] 
34. Branderhorst W, Vastenhouw B, Beekman FJ (2010) Pixel-based subsets for rapid multi-pinhole SPECT reconstruction. Phys Med Biol 55: 2023-2034. [Crossref]

35. Wu C, van der Have F, Vastenhouw B, Dierckx RA, Paans AM, et al. (2010) Absolute quantitative total-body small-animal SPECT with focusing pinholes. Eur J Nucl Med Mol Imaging 37: 2127-2135. [Crossref]

36. Ogawa K, Harata Y, Ichihara T, Kubo A, Hashimoto S (1991) A practical method for position-dependent Compton-scatter correction in single photon emission CT. IEEE Trans Med Imaging 10: 408-412. [Crossref]

37. Van Lent PL, Blom A, Holthuysen AE, Jacobs CW, Van De Putte LB, et al. (1997) Monocytes/macrophages rather than PMN are involved in early cartilage degradation in cationic immune complex arthritis in mice. J Leukoc Biol 61: 267-278. [Crossref]

38. Choi MC, Choi WH (2018) Mithramycin A Alleviates Osteoarthritic Cartilage Destruction by Inhibiting HIF-2alpha Expression. Int J Mol Sci 19: 1411. [Crossref]

39. Song JL, Li L, Fang H, Cai DZ (2018) Intraperitoneal injection of thalidomide alleviates early osteoarthritis development by suppressing vascular endothelial growth factor expression in mice. Mol Med Rep 18: 571-579. [Crossref]

40. Holyoak DT, Tian YF, van der Meulen MC, Singh A (2016) Osteoarthritis: Pathology, Mouse Models, and Nanoparticle Injectable Systems for Targeted Treatment. Ann Biomed Eng 44: 2062-2075. [Crossref]
41. Smith MD (2011) The normal synovium. Open Rheumatol J 5:100-106. [Crossref]

42. Kennedy A, Fearon U, Veale DJ, Godson C (2011) Macrophages in Synovial Inflammation. Front Immunol 2: 52. [Crossref]

43. Kinne RW, Brauer R, Stuhlmuller B, Palombo-Kinne E, Burmester GR (2000) Macrophages in rheumatoid arthritis. Arthritis Res 2: 189-202. [Crossref]

44. Udalova IA, Mantovani A, Feldmann M (2016) Macrophage heterogeneity in the context of rheumatoid arthritis. Nat Rev Rheumatol 12: 472-485. [Crossref]

45. Put S, Westhovens R, Lahoutte T, Matthys P (2014) Molecular imaging of rheumatoid arthritis: emerging markers, tools, and techniques. Arthritis Res Ther 16: 208.

46. Winkel LC, Groen HC, van Thiel BS, Muller C, van der Steen AF, et al. (2014) Folate receptor-targeted single-photon emission computed tomography/computed tomography to detect activated macrophages in atherosclerosis: can it distinguish vulnerable from stable atherosclerotic plaques? Mol Imaging 13. [Crossref]

47. Varasteh Z, Hyafil F, Anizan N, Diallo D, Aid-Launais R, et al. (2017) Targeting mannose receptor expression on macrophages in atherosclerotic plaques of apolipoprotein E-knockout mice using (111)In-tilmanocept. EJNMMI Res 7: 40. [Crossref] 